\title{
A Behavioral Perspective on Vietnam's Corporate Safety Measures and Nudges in COVID-19
}

\author{
Bui Gia Han Pham ${ }^{1}$ and Philip Liang\# \\ ${ }^{1}$ British International School Hanoi, Vietnam \\ \#Advisor
}

ABSTRACT

Robust enforcement of safety procedures is of critical importance for businesses in Vietnam in their efforts to prepare employees for a return to physical workplaces, especially in a country with only $38.8 \%$ of its population fully vaccinated as of late November 2021 (Ritchie et al., 2020). In academic literature, nudges are traditionally believed to enhance compliance to protective measures (Debnath and Bardhan, 2020). The aim of this paper is to test the strength of such theoretical analysis of nudges' effectiveness by applying it to the context of companies in Vietnam. We performed a survey of 41 companies in various sectors and used regression models to interpret our data. One study found a positive significant relationship between automatic registration, monetary rewards, and percentage of employees vaccinated. In the same study, we found an average increase in vaccine uptake of $27.7 \%$ to $45.6 \%$ due to vaccination nudges. In our second study, we found no evidence that sanitising nudges encourage employees' usage of sanitary utensils provided to them. Although our small sample size was a major limitation of this study, our findings put the functionality of nudges into a developing country's context and analysed socio-economic factors that might have caused our results to differ from prior research. With this study being the first to examine the use of nudges to promote pro-health behaviour in large corporations during COVID-19, future research could delve deeper into the effect of other non-mandatory safety measures on overall workplace health.

\section{Introduction}

Over the past year, the COVID-19 pandemic has transformed workplaces. As of October 11, 2021, more than 55 large corporations are permanently embracing hybrid, remote-first, or fully remote work for their employees (Henry et al., 2021). Additionally, according to Global Workplace Analytics, it is estimated that by the end of $2021,25 \%-30 \%$ of employees in the U.S. would be working from home (Lister, 2021). In Vietnam specifically, there are a few large corporations that are reportedly implementing a work-from-home model for their employees, such as FPT Joint Stock Company, Tiki, Be Group (Hoang, 2021).

Despite this apparent move to digital work in certain companies, the vast majority of corporations in Vietnam may still be in the process of experimenting with different ways to implement remote work in their organisations. Thus, keeping their physical workplaces Covid-free is still considered to be one of their priorities.

Out of 41 companies surveyed in this study, we found that $64.1 \%$ of firms reported that their biggest challenge in devising effective COVID-19 measures is convincing employees to take recommended, but not mandatory measures. This behavioural problem is evident in a study of 6000 Vietnamese students that documents that among students, there is a $16.59 \%$ vaccine hesitancy rate (Khuc et al., 2021). Other frictions, such as inertia or procrastination, may also prevent employees from following through with many safety guidelines (Bertrand, Mullainathan, and Shafir 2006). As a result, effective corporate safety measures should aim to eliminate the effects of psychological friction by making it easier for employees to proactively practice health-conscious behaviours during COVID-19, which can be achieved through a concept called nudging. 
This paper will explore the efficacy of these nudges in maximising safety in businesses in Vietnam during COVID-19. After evaluating relevant literature, this paper examines two hypotheses, namely that vaccination nudges increase vaccine uptake and sanitising nudges increase frequency of usage of sanitary tools. It then tests them based on survey data collected from Vietnamese companies. The last section of this paper discusses the implications of the findings relative to the local context and suggests recommendations for future research.

\section{Literature Review}

This literature review will look at existing safety measures that have been adopted by companies during COVID-19, as well as nudges that are not studied by this paper but can potentially be helpful for future research to investigate.

\section{COVID-19 Corporate Safety Measures}

There has been robust research on viable Covid safety solutions in the hospitality and service industry. Gursoy and Chi (2020) observed that visible sanitising and social distancing efforts, such as hand sanitisers at the entry, staff wearing masks, or technology adoption like contactless payment, had the most important influence on the willingness of customers to eat out at restaurants. Hu et al. (2021) and Dennerlein et al. (2020) also found several similar factors that lead to higher compliance, including ongoing promotion of the importance of health in a pandemic, increased involvement and prioritisation of employees' wellbeing in protective strategies.

Perhaps the most common approach by businesses to ensure safety is temperature screening. This has proven to be limited in its effectiveness, since with the new variants of Covid and their shifting ways of affecting the body and causing symptoms, many of those who go over the mark of a certain thermal temperature value are not Covidpositive, while those who are tested positive may still feel well enough to go to work on the day of screening (Bielecki et al., 2020, p.2). Temperature testing also fails to identify asymptomatic or pre-symptomatic people who do transmit Covid without developing any symptoms. Data collected from the same study suggest that, at best, $44 \%$ of cases could be detected using body temperature measurements during exit (Bielecki et al., 2020, p.1). More alarmingly, Stave et al. (2021), in their survey of 20 medical directors at large multinational companies from February 2020 to March 2021, discovered that more than 2000 cases of Covid in these workplaces were not identified through screening. This equates to, on average, only one identified case of COVID-19 through temperature screening out of 40 cases that were missed (Stave et al., 2021, p.2)

The findings of these studies, although they did advance the knowledge of common safety strategies in industries, did not consider external factors that can affect an individual's long-term compliance with these safety procedures. In other words, the frameworks studied may increase the motivation to take up these procedures but fail to guarantee the outcome of such motivation, where employees will follow thoroughly and consistently with these measures. This paper aims to fill in this knowledge gap, by examining ways that behavioural interventions such as nudges can boost health outcomes and remove barriers to following through on these protective measures.

\section{Nudges}

Nudges are interventions that alter 'people's behavior in a predictable way without forbidding any options or significantly changing economic incentives' (Thaler and Sunstein, 2009). Within public health settings, nudges have been shown to be effective in promoting prosocial choices such as organ donation (Davidai et al., 2012) and influenza vaccination (Thaler and Sunstein, 2009). In COVID-19, nudges have been cemented in various policies adopted around the world, such as optimal contact tracing through a default system of filling in contacts (Beidas et al., 2020), informational nudges on the dangers of infection (Blackman and Hoffman, 2021). The following nudges that will be analysed in-depth would potentially be effective in ensuring workplace safety and thus could be tested by future research. 


\section{Text-based reminders}

Text-message nudges are perhaps most commonly studied in current literature. A large-scale flu vaccination study conducted by the University of Pennsylvania's Behavior Change for Good Initiative revealed that sending a text message to individuals stating that the vaccine is reserved in their name led to a significant increase in uptake (Milkman et al., 2021). Dai and Saccardo (2021) also found that reminders containing ownership language such as 'Vaccine $X$ has just been made available for you' or 'Claim your dose', is reported to have the largest effect on the number of vaccine appointments, increasing the rates in 6 days by $94.84 \%$. This phenomenon can be explained through the endowment effect, which states that individuals are keener on utilising or holding on to things that belong to them.

\section{Visual Cues}

A signage system may be a helpful way to drive effective public messaging. An important consideration in choosing which signs to display in workplaces is whether they embed messages that are receptive and motivating for employees during a stress-inducing pandemic (Shaw et al., 2020).

As such, signs that emphasise collective belonging and communal solidarity have been proven to be effective in encouraging compliance. This sentiment has been mainly used in national campaigns that encourage people to stay at home to protect themselves and their loved ones, with the saying "We are all in this together". An example of this in the corporate context is "Keeping our spaces healthy, together.", a slogan at WeWork, an American real estate company that designs and builds physical shared office spaces (Mannarino et al., 2021). Ceylan and Hayran (2021) surveyed 119 students and found that prosocial messages using the pronoun 'our' ('For all our health, stay at home') created a higher feeling of joint responsibility to protect from coronavirus than the self-interested message with the pronoun 'your' ('For your own health'). Such a feeling of collective obligation may help solve the collective action problem where an individual is reluctant to make their own sacrifices for public interest, such as wearing a mask or practicing social distancing, unless convinced otherwise (Harring et al., 2020).

Similarly, companies can subtly establish communal spirit through reinforcing the company's culture or motto, by using the same theme or colour of the company's brand image in designing the cues or incorporating a symbol or mascot that represents the company. The outcome of this strategy is an enhancement in familiarity and thus trust among employees, which is found to be a crucial determinant in ensuring compliance with public policies (Marien and Hooghe, 2011).

\section{Coloured Wristbands}

To ensure effective social distancing, it is important to first understand how each person wants to be interacted with. In tackling the challenges of deciphering suitable forms of interactions, enterprises such as Social Bands are spearheading the creation of wristbands that give visual indicators of one's comfort level in social circles according to the visible colours. This product is reported to have been utilised by businesses and schools alike in approximately 50 countries (PRNewswire, 2021). Each color signals a different level of caution when interacting, with red, yellow, and green indicating no direct contact, indirect contact, and more direct contact respectively (McMillin, 2020). With heterogeneity in vulnerability during COVID-19, this allows employees, especially high-risk individuals, to feel safer when they have control over how they want to be interacted with.

Despite these findings, there are still some limits to the current state of research in the field. The majority of these studies evaluate common nudges exclusively in one context or environment, for example, public health policies, influenza vaccination, or organ donation, instead of examining ways in which these nudges can be cross implemented in other settings, such as corporate workplaces. This paper will focus on looking at the effects of automatic registration, monetary rewards on vaccination uptake and sanitising nudges on frequency of usage by employees in businesses.

\section{Hypotheses}


For this study, two hypotheses were developed. Hypothesis 1 looks at the effectiveness of two vaccination nudges (automatic registration and monetary rewards) in encouraging vaccine uptake, which corresponds to the aim of Study 1. Hypothesis 2 focuses on the relationship between sanitising nudges (placement of hand sanitisers and provision of masks in shared spaces) and frequency of usage.

Hypothesis 1: Vaccination nudges, namely default system of automatic vaccine registration and monetary rewards increase the rate of vaccine uptake, with monetary rewards to a lesser extent.

This hypothesis was mainly based on existing literature. A major pattern seen across research on public health policies is how vaccine intentions do not always reflect actual vaccine uptake (Sheeran, 2002). One of the ways individual behaviour can cause this lag in intention and uptake is inertia, or aversion to 'hassle costs' (Bertrand et al., 2006), which occurs when an individual is reluctant to go through the whole process of registration to scheduling and confirmation. This may be because it is long, or because they are unsure of the rules (Liebman and Luttmer, 2015).

Methods to remove such barriers include a default or opt-out system and onsite vaccination. The default system of vaccination is defined as preset courses of action that take effect if nothing is specified by the decisionmaker (Thaler and Sunstein, 2009). In other words, companies may automatically register all of their employees for vaccine appointments, so that they do not have to spend time going through the sophisticated process with little support or information. For those who wish to not be registered for appointments, they are free to cancel or deny the appointment with no harm associated with that decision. This subset of the population, however, is reported to be the minority of cases, since people are prone to conform to the status quo, in this case, the default registration for vaccine appointments. This has been proven to be the case in Davidai's 2012 study of participants from the United States and Germany, where they found that an opt-out system that automatically registers people as organ donors increases organ donations (Davidai et al., 2012).

A second incentive structure that encourages employees to take vaccines is monetary rewards, a strategy that has been adopted by some companies in the United States and Germany, where workers received one-time payments for getting vaccinated (Maruf, 2021). Contrary to expectations of a positive correlation between monetary rewards and vaccination intentions, extensive research has found that monetary incentives for pro-social or morally good behaviour such as vaccination to protect oneself and the surrounding community may erode intrinsic motivation, since commodifying good behaviour may make the act of doing it lose its inherent moral value (Largent and Miller, 2021). In the context of COVID-19, paying people for COVID-19 vaccinations may signal that vaccination is undesirable or even dangerous. In fact, an empirical study involving residents in the United States found that $70 \%$ of participants take vaccinations even without compensation while paying them $\$ 20$ reduces the number of people who are willing to do so (Serra-Garcia and Szech, 2021).

In the same study, exceptions to such cases are when the amount of payment is significantly higher, such as $\$ 100$. These monetary incentives increase motivation to take vaccines by weighing out against the commodification effects. Thus, in considering whether to give out monetary rewards for employees to take vaccines, companies should factor in the cost-effectiveness of such measures - given that the majority of individuals are intrinsically motivated to opt into prosocial acts, the only subset that such a high amount of payment actually benefits is considerably small.

\section{Hypothesis 2: Placement of hand sanitisers in shared spaces and Provision of masks encourages usage from employees}

Investing in alcohol dispensers to place around shared spaces in workplaces, such as doorknobs, printers, and photocopiers can be an extremely cost-effective way for companies to increase sanitation rates among employees. This framework arranges choices in a way that is the most straightforward and convenient for employees, such that they 
do not have to carry a sanitiser around and can still keep safe. Notably, placing dispensers in these places subconsciously acts as a reminder for people to use the sanitisers. This effectively removes action bias, where people are inclined to inaction, by being a constant visual encouragement to disinfect. Moreover, people are found to follow what the person before them does, especially in public space, as a result of herd mentality, which means, if there is a person who uses the hand sanitiser, others tend to do the same action.

\section{Methodology}

The sample of this study consists of 41 companies in Vietnam in a range of sectors: manufacturing, retail, to trading and services. An invitation email to participate in the study was sent to the addresses of employees in a convenience sample of 45 companies in total, which contains a link to an online survey of Qualtrics. Companies who responded that they agreed to take part in the study fill out the form (response rate $=91.1 \%$ ). The aim of the survey is to understand the frequency and effectiveness of measures that companies use to increase safety in their workplaces during COVID-19. Questions are mostly multiple choice, concerning the presence and compliance levels of employees with safety measures of sanitising and vaccination.

We implemented 2 separate studies. Study 1 explores the relationship between automatic registration and monetary rewards in nudging vaccination uptake by employees. Study 2 analyses the relationship between the provision of masks and placement of hand sanitisers in shared spaces on frequency of usage.

\section{Vaccination Nudges}

\section{Multivariate Linear Regression Model}

Study 1 was designed to find the combination of variables, including nudges related to vaccination and percentage of employees vaccinated, that has the strongest correlation with one another. To achieve this, participants were asked in a multiple choice question to select measures that are implemented in their companies out of all 5 safety measures (Onsite Vaccination, Automatic Registration, Monetary Rewards, Reminders with name, Others, which gives companies choice to indicate a procedure that is not mentioned in the provided answers). Companies also indicated at the beginning the percentage of employees that are vaccinated with a second dose. In the data that we were given, most values of percentage vaccinated fall between 0.7 to 0.9 . In the following analysis, we restrict our attention to the 40 larger companies with 400 or more employees that responded to our survey. A multivariate linear regression model was first performed, which included the percentage of employees vaccinated as a dependent variable and all other variables as independent. We then conducted an ablation study in which we systematically extracted independent variables and examined the strength of the relationship between variables in the subsequent combinations.

\section{t-Test}

The following questions were posed: 'What is the percentage of employees that are vaccinated in your company?' and 'If your company did nothing to encourage vaccination, what do you think the vaccination rate (second dose) among your employees would be? (\% of total employees)'. A few companies indicated their answers in a range; thus, we used low-effect and high-effect assumptions in an unequal variances t-test to calculate the mean percentage of vaccinated employees. In addition, we computed $95 \%$ confidence intervals for the vaccination rate with and without the company's efforts.

\section{Sanitising Nudges}

In Study 2, we examined the frequency of usage of sanitary tools when they are being provided in the common area of workplaces. We asked participants to indicate what sanitising measures their companies implement from the choices provided, including 'Placement of hand sanitisers in shared spaces' and 'Provision of masks/ sanitising spray/ wipes 
in shared spaces'. Respondents were then asked to state the frequency of employees' usage of these utensils from a scale of 'Sometimes', 'Frequently', to 'Very Frequently'.

To interpret the results, we performed an ordinal logistics regression model, in which we assigned ordinal values to the responses using a 3-point scale, with 0 being 'Sometimes', 1 being 'Frequently', and 2 being 'Very Frequently'. The dependent variables were indicator variables for whether companies implemented 'Placement of hand sanitisers in shared spaces' and 'Provision of masks/ sanitising spray/ wipes in shared spaces'.

\section{Results}

\section{Vaccination Nudges}

We found that out of all combinations of variables, the strongest linear correlation and a statistically significant relationship exists between vaccination registration, monetary rewards (which range from \$20 to \$200) and percent of employees vaccinated. For this combination, we derived an adjusted R squared value of 0.286 and the p-value of 0.002 (See Table 1). Our results suggest that a monetary reward of $\$ 20$ produces a much stronger effect on vaccination than automatic registration.

Table 1. Relationship Between Vaccination and Automatic Registration and Monetary Rewards

\begin{tabular}{lllll} 
& Estimate & SE & t-value & $\operatorname{Pr}(>|\mathrm{t}|)$ \\
\hline Intercept & 0.82833 & 0.03041 & 27.236 & $<2 \mathrm{e}-16$ \\
Automatic Registration & 0.14881 & 0.03813 & 3.903 & 0.000459 \\
Monetary Rewards & 0.14167 & 0.08047 & 1.761 & 0.087869 \\
& & & & \\
Multiple R-squared & Adjusted R-squared & & F-statistic & p-value \\
\hline 0.3279 & 0.2859 & & 7.805 & 0.001735
\end{tabular}

In terms of our $t$-test on perceived effectiveness of vaccine nudges, we found that, on average, implementing measures to encourage vaccination will result in $85 \%$ of their employees vaccinated, while only $50 \%$ are vaccinated when no efforts are in place. We are $95 \%$ confident that the average vaccination rate of Vietnamese companies after the implementation of these measures is from $79.27 \%$ to $91.85 \%$ of total employees, while the percentage of employees vaccinated with no efforts is $40.66 \%$. The average perceived increase in vaccination uptake after the measures are in place is at least $27.73 \%$ to $45.62 \%$.

\section{Sanitising Nudges}

From our model, we obtained t-values of $-0.16,0.753,-0.884$, and 1.337 (See Table 2). These small t-scores suggest the absence of a strong link between our data sets. In other words, we found no evidence of a significant relationship between placement of hand sanitisers in shared spaces, provision of masks, and reported frequency of usage.

Table 2. Relationship between Placement of sanitisers and Provision of masks in shared spaces and Frequency of Usage

Value $\quad$ Std. Error t-value




\author{
Placement of Sanitiser \\ Provision of masks \\ Sometimes | Frequently \\ Frequently | Very frequently
}

$\begin{array}{rr}-0.2255844 & 1.4101851 \\ 0.6845998 & 0.9093491 \\ -1.2557448 & 1.4199284 \\ 1.9403804 & 1.4511669\end{array}$

$-0.1599679$

0.7528459

$-0.8843719$

1.3371174

\section{Discussions and Limitations}

As with the majority of studies, the design of the current study is subject to several broad limitations. Firstly, to the authors' knowledge, this is the first paper that examines the functionality of companies' use of nudges in Covid safety measures. Secondly, the relatively small sample size of this paper might have reduced analytical power and ability to generalise our findings to all companies in Vietnam. The third limitation concerns the way data was collected. Due to the absence of a randomised controlled experiment that can investigate the effects of one safety measure on overall infection rates or vaccination rates, our study could only infer correlation between these variables. We also were not able to single out one safety measure that is the most effective out of all that are discussed in this paper, as it can be likely that all variables contribute some effect on compliance, uptake, or frequency of use, but we are unaware of which effect is larger. Future research could immensely benefit from designing a controlled experiment to determine a causal nature of the relationship between these safety measures.

\section{Vaccination Nudges}

\section{Automatic Registration and Monetary Rewards}

Scientists look at both $\mathrm{R}$ squared and p-value when trying to confirm the strength of the model. Despite the relatively low R squared score, it does not suggest a lack of relationship between the independent variables and the percentage of employees vaccinated. In particular, our R-squared value informs us that a little more than a quarter of the variability in our data points about the regression line can be explained by the model itself. Although this equates to a limitation in our study regarding perfectly understanding the relationships presented by the model, our small p-value of 0.002 helps corroborate that the relationship identified between the independent and dependent variables is statistically significant. Thus, we can trust that there is in fact a relationship between vaccination registration, monetary rewards, and percentage of employees vaccinated, aligned with our hypothesis.

It is worth discussing the implications of our R-squared value. Firstly, our low R-squared score is reasonably expected as we did not have perfect knowledge on individual employees' background, vulnerability, or other factors that may cause unpredictable changes in behaviour in a pandemic. As this may have influenced our inability to explain variability in our data points, future research could take into consideration the relevance of collecting such personal information from employees.

Secondly, the uncertainty of the relationships denoted by our R-squared score may indicate the presence of variables other than the nudges studied affecting the percentage of employees vaccinated . As a developing country, vaccine production has been limited, with stark shortage of vaccines happening in the country (Anh and Loc, 2021). As of November 17th, 2021, only $38.8 \%$ of the population is fully vaccinated. It is also important to note that healthcare infrastructure in Vietnam is severely underdeveloped, suffering from limited capacity and inadequate healthcare equipment (World Bank Group, 2021). Therefore, this context of vaccine supply in Vietnam may lead to high consciousness among employees to get vaccinated to protect themselves. Such high intrinsic motivation could potentially be the variable that affects vaccination percentage, as opposed to the nudges in question that were assumed to have an effect. Additionally, in the response section to 'Others', some companies indicated they educated their employees about the importance and safety of vaccines and regularly published vaccination status of employees, which corroborates the findings of Khuc et al. (2021) suggesting lack of information on vaccines as one of the reasons for 
vaccine hesitancy. It would be helpful for future studies to discuss the strengths of these different approaches in enhancing workplace safety.

In terms of our significant p-value, our findings add to the results of other research that study the effectiveness of a default system on encouraging influenza vaccination uptake (Chapman et al., 2010) and organ donations (Davidai et al., 2012) by confirming the relationship between the default system and vaccination rate of employees. Future research could look more into the effects of automatic registration on solely vaccine-hesitant employee populations, potentially through questionnaires, to find a more significant relationship. Our method of documenting the strongest correlation between a combination of variables can be replicated in future studies that cannot afford to design a controlled experiment on human behaviour.

Our results support the idea that monetary rewards can encourage vaccination. There is a possibility that monetary rewards could erode the moral value of getting vaccinated to benefit communal interests (Loewenstein and Cryder, 2020; Serra-Garcia and Szech, 2021). Given the vaccine shortage, it is unclear whether Vietnamese workers would view getting vaccinated as a prosocial act. It is plausible that employees only perceive private benefits to vaccination because they believe that requesting vaccines for themselves will deny access to vaccination to another person. In order for monetary rewards to discourage vaccination, two conditions must be met: intrinsic motivation must exist and be eroded by monetary rewards and monetary rewards must influence behavior less than the intrinsic motivation they destroyed.

Surprisingly, we estimate that monetary compensation causes a much larger increase in vaccination rates than automatic enrollment. To an extent, this finding challenges our hypothesis and the conventional concern that monetary rewards erode intrinsic motivation for morally significant acts when the amount provided is too small. This difference may be traced to the local context of our study. The average price level of goods in Vietnam is approximately 0.34 US Dollars, which means the factor of the income that the monetary rewards accounts for is different from that of studies looking at the United States. This may incur an effect on the real significance of these compensations, which manipulates Vietnamese employees' behaviours in other ways that previous research findings cannot generalise. The limitation of this study is that the causal effects of low and high compensation are not studied separately, which means it is uncertain as to whether the high compensation is causing an effect, or the low compensation.

\section{Perceived Effectiveness of Vaccination Nudges}

The main takeaway from our data is that companies' vaccination efforts are perceived by their employees to be substantially effective in encouraging vaccination uptake. This confirms our hypothesis that overall vaccination nudges have an effect on motivating or easing the process for employees to get vaccinated. The limitation of this study is that we did not test for the 'real' effectiveness of vaccination nudges, but rather their perceived value in increasing vaccine uptake.

\section{Sanitising Nudges}

Our research suggests no evidence of a relationship between sanitising nudges, namely placement of hand sanitisers and provision of masks in public spaces and reported frequency of usage by employees, which challenges our hypothesis. This finding, however, aligns with previous research which found no effect of nudges on intentions to wear masks (Dimant et al., 2021). Additionally, it might be possible that employees already brought their own masks and hand sanitisers to workplaces, which means there is less of a strong need to use utensils provided by their employers.

\section{Conclusion}

This paper contributes to a growing body of literature examining the effect of behavioural interventions on encouraging protective measures by investigating the application of nudges in large corporate settings on employees' vaccination uptake and compliance rate. The main takeaways from our study are three-fold: firstly, we found a relationship 
between automatic registration, monetary rewards, and percentage of employees vaccinated. Secondly, vaccination nudges are believed to have increased vaccination uptake by a large percentage. Thirdly, there is no evidence for a correlation between sanitising nudges and frequency of usage. Our results, when compared to prior research findings, pointed out the possibility of other factors in the context of Vietnam, such as intrinsic motivation or education, causing an effect on overall compliance. Thus, given the changing behaviours of employees that correspond to the unforeseen complications of the pandemic, it is pertinent that our research could be extended upon to deepen the understanding of nudges' functionalities in encouraging healthy behaviour across different settings and contexts.

\section{Acknowledgments}

I would like to thank my advisor, Philip Liang, for their mentorship and guidance throughout the development of this research paper. I also wish to extend my gratitude to Tyler Moulton, Sara Ashbaugh, Prakriti Sharma, and other anonymous reviewers for their kind support in reviewing my work. Lastly, I want to thank Lumiere's Education's managers, Dhruva Bhat and Stephen Turban, for providing me with this opportunity to explore the field of research.

\section{References}

Anh and Loc. (2021). Cơ Hội Nào Cho Vắc Xin Việt? TUOI TRE ONLINE. Retrieved November 29, 2021, from https://tuoitre.vn/co-hoi-nao-cho-vac-xin-viet-20211107083638365.htm.

Beidas, R. S., Buttenheim, A. M., Feuerstein-Simon, R., Kilaru, A. S., Asch, D. A., Volpp, K. G., Lawman, H. G., and Cannuscio, C. C. (n.d.). Optimizing and implementing contact tracing through behavioral economics. NEJM Catalyst Innovations in Care Delivery. Retrieved November 29, 2021, from https://catalyst.nejm.org/doi/full/10.1056/CAT.20.0317.

Bertrand, M., Mullainathan, S., and Shafir, E. (2006). Behavioral Economics and Marketing in Aid of Decision Making among the Poor. Journal of Public Policy and Marketing, 25(1), 8-23. https://doi.org/10.1509/jppm.25.1.8

Bielecki, M., Crameri, G., Schlagenhauf, P., Buehrer, T. W., and Deuel, J. W. (2020). Body temperature screening to identify SARS-CoV-2 infected young adult travellers is ineffective. Travel medicine and infectious disease, 37, 101832. https://doi.org/10.1016/j.tmaid.2020.101832

Blackman, A., and Hoffmann, B. (2021). Research insights: Can informational nudges increase compliance with COVID-19 measures? https://doi.org/10.18235/0003282

Ceylan, M., and Hayran, C. (2021). Message Framing Effects on Individuals' Social Distancing and Helping Behavior During the COVID-19 Pandemic. Frontiers in psychology, 12, 579164. https://doi.org/10.3389/fpsyg.2021.579164

Chapman, G. B., Li, M., Colby, H., and Yoon, H. (2010). Opting in VS opting out of influenza vaccination. JAMA, 304(1), 43. https://doi.org/10.1001/jama.2010.892

Dai, H., Saccardo, S., Han, M. A., Roh, L., Raja, N., Vangala, S., Modi, H., Pandya, S., and Croymans, D. M. (2021). Behavioral nudges increase covid-19 vaccinations: Two randomized controlled trials. https://doi.org/10.1101/2021.04.12.21254876 
Davidai, S., Gilovich, T., and Ross, L. D. (2012). The meaning of default options for potential organ donors. Proceedings of the National Academy of Sciences, 109(38), 15201-15205. https://doi.org/10.1073/pnas.1211695109

Debnath, R., and Bardhan, R. (2020). India nudges to contain COVID-19 pandemic: A reactive public policy analysis using machine-learning based topic modelling. PloS one, 15(9), e0238972. https://doi.org/10.1371/journal.pone.0238972

Dennerlein, J. T., Burke, L., Sabbath, E. L., Williams, J. A. R., Peters, S. E., Wallace, L., Karapanos, M., and Sorensen, G. (2020). An Integrative Total Worker Health Framework for Keeping Workers Safe and Healthy During the COVID-19 Pandemic. Human Factors, 62(5), 689-696. https://doi.org/10.1177/0018720820932699

Dimant, E., Pieper, D., Clemente, E. G., Dreber, A., \&amp; Gelfand, M. J. (2021). Politicizing mask-wearing: Predicting the success of behavioral interventions among Republicans and Democrats. SSRN Electronic Journal. https://doi.org/10.2139/ssrn.3915256

Gursoy, D., and Chi, C. G. (2020). Effects of covid-19 pandemic on hospitality industry: Review of the current situations and a research agenda. Journal of Hospitality Marketing and Management, 29(5), 527-529. https://doi.org/10.1080/19368623.2020.1788231

Harring, N., Jagers, S. C., \&amp; Löfgren, Å. (2021). Covid-19: Large-scale collective action, government intervention, and the importance of trust. World Development, 138, 105236. https://doi.org/10.1016/j.worlddev.2020.105236

Henry and Remote work 2021 and the Future of Remote Work (2021). Every company going remote permanently: Oct 11, 2021 update. Build a better company. From home. Retrieved November 29, 2021, from https://buildremote.co/companies/companies-going-remote-permanently/.

Hoang, N. (2021). Remote Working: The Future is now. RMIT University. Retrieved November 29, 2021, from https://www.rmit.edu.vn/news/all-news/2020/apr/remote-working--the-future-is-now.

Hu, X., Yan, H., Casey, T., and Wu, C.-H. (2021). Creating a safe haven during the crisis: How organizations can achieve deep compliance with covid-19 safety measures in the hospitality industry. International Journal of Hospitality Management, 92, 102662. https://doi.org/10.1016/j.ijhm.2020.102662

Khuc, Quy V., Trang Nguyen, Thuy Nguyen, Linh Pham, Dang-Trung Le, Hong-Hai Ho, Tien-Binh Truong, and Quoc-Khai Tran. (2021). Young Adults' Intentions and Rationales for COVID-19 Vaccination Participation: Evidence from a Student Survey in Ho Chi Minh City, Vietnam. Vaccines 9, no. 7: 794. https://doi.org/10.3390/vaccines9070794

Largent, E. A., and Miller, F. G. (2021). Problems with paying people to be vaccinated against COVID-19. JAMA, 325(6), 534. https://doi.org/10.1001/jama.2020.27121

Lister, K. (2021). Work-at-Home After Covid-19 - Our Forecast. Global Workplace Analytics. Retrieved November 27, 2021, from https://globalworkplaceanalytics.com/work-at-home-after-covid-19-our-forecast. 
Loewenstein, G., and Cryder, C. (2020). Why paying people to be vaccinated could backfire. The New York Times. Retrieved November 24, 2021, from https://www.nytimes.com/2020/12/14/upshot/covid-vaccinepayment.html.

Mannarino, M., Cvetkovic, A., and Dermott, A. (2021). Signs that reinforce social distancing in the Office. Ideas. Retrieved November 27, 2021, from https://www.wework.com/ideas/workspace-solutions/flexibleproducts/signs-that-reinforce-social-distancing-in-the-office.

Marien, S., and Hooghe, M. (2011). Does political trust matter? an empirical investigation into the relation between Political Trust and support for law compliance. European Journal of Political Research, 50(2), 267-291. https://doi.org/10.1111/j.1475-6765.2010.01930.x

Maruf, R. (2021). "These companies are paying their employees to receive the Covid-19 vaccine." Retrieved July 20, 2021, from https://edition.cnn.com/2021/03/24/business/covid-vaccine-incentivescompanies/index.html

McMillin, D. (2020). Social Distancing Solution: Wristbands do the talking for attendees. PCMA. Retrieved November 29, 2021, from https://www.pcma.org/social-distancing-solution-wristbands-attendees/.

Milkman, K. L., Patel, M. S., Gandhi, L., Graci, H. N., Gromet, D. M., Ho, H., . .Duckworth, A. L. (2021). A megastudy of text-based nudges encouraging patients to get vaccinated at an upcoming doctor's appointment. Proceedings of the National Academy of Sciences of the United States of America, 118(20). https://doi.org/10.1073/pnas.2101165118

Ritchie, H., Mathieu, E., Rodés-Guirao, L., Appel, C., Giattino, C., Ortiz-Ospina, E., Hasell, J., Macdonald, B., Beltekian, D., \&amp; Roser, M. (2020, March 5). Coronavirus (COVID-19) vaccinations - statistics and research. Our World in Data. Retrieved November 29, 2021, from https://ourworldindata.org/covidvaccinations? country $=\sim \mathrm{VNM}$.

Serra-Garcia, M., and Szech, N. (2021). Choice architecture and incentives increase COVID-19 vaccine intentions and test demand. SSRN Electronic Journal. https://doi.org/10.2139/ssrn.3827616

Shaw, W. S., Main, C. J., Findley, P. A., Collie, A., Kristman, V. L., and Gross, D. P. (2020). Opening the workplace after covid-19: What lessons can be learned from return-to-work research? Journal of Occupational Rehabilitation, 30(3), 299-302. https://doi.org/10.1007/s10926-020-09908-9

Social Bands, L. L. C. (2021). Social distancing bracelets program proves effective worldwide. Social Distancing Bracelets Program Proves Effective Worldwide. Retrieved November 29, 2021, from https://www.prnewswire.com/news-releases/social-distancing-bracelets-program-proves-effectiveworldwide-301409196.html.

Stave, G. M., Smith, S. E., Hymel, P. A., and Heron, R. (2021). "Worksite Temperature Screening for COVID-19." Journal of occupational and environmental medicine, 63(8), 638-641. https://doi.org/10.1097/JOM.0000000000002245

Thaler, R. H., and Sunstein, C. R. (2009). "Nudge: Improving decisions about health, wealth and happiness." London: Penguin Books Ltd. 
World Bank Group. (2021). A world bank-financed project helps improve the public health service delivery in the northern part of Vietnam, benefiting millions of people. World Bank. Retrieved November 29, 2021, from https://www.worldbank.org/en/news/feature/2021/03/01/vietnams-rural-populace-enjoys-better-healthcareservices. 